\title{
Solutions for Torque and Speed Measurement on Electric Machine Controllers Test Benches
}

\section{Soluciones para la medición de par y velocidad en bancos de ensayos de controladores de máquinas eléctricas}

\author{
A. Veyrat Durbex ${ }^{{ }^{*} 1}$, Y. Nachajon Schwartz ${ }^{\dagger 2}$, H. Tacca ${ }^{\dagger 3}$ \\ † Laboratorio de Control de Accionamientos Tracción y Potencia (LABCATYP), Departamento de Electrónica, Facultad de \\ Ingeniería, Universidad de Buenos Aires, Paseo Colón 850, C1063ACV, Buenos Aires, Argentina

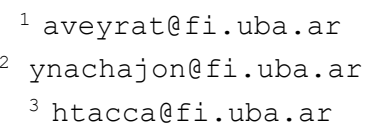 \\ * Departamento de Energía, Facultad de Ingeniería, Universidad de Buenos Aires \\ Paseo Colón 850, C1063ACV, Buenos Aires, Argentina
}

Recibido: 06/04/21; Aceptado: 08/06/21

\begin{abstract}
The continuous search for improvement in electromechanical developments requires a clear understanding of the torque measurement required in each application. The decisions made will have a profound impact on the quality and cost of the results. This article presents the principles normally used for sensing mechanical torque on a shaft and the reasons for the widespread use of strain gauges. The importance of differentiation between static and dynamic measurements and the current technologies used in each case are discussed. The relevant factors that define a transducer are described and a comparative analysis between them is carried out, to then examine the possible mounting methods, with their benefits and limitations. Finally, different low-cost solutions are proposed for the design of the torque and speed measurement section without sacrificing system performance, for different test benches for electrical machine controllers, including the necessary signal conditioning electronics.

The article aims to be a tutorial compendium of topics to study to successfully implement a test bench without damaging the torque transducer or introducing measurement errors, since the information on these topics is scattered and it is difficult to access knowledge of the selection criteria and project procedures.
\end{abstract}

Keywords: Electric machines; torque transducer; torque measurement.

Resumen - La continua búsqueda de la mejora en los desarrollos electromecánicos impone una clara comprensión de la medición de par necesaria en cada aplicación. Las decisiones tomadas tendrán un profundo impacto en la calidad y el costo de los resultados. En este artículo se presentan los principios comúnmente empleados para el sensado de par mecánico en un eje y el porqué de la utilización extendida de las galgas extensiométricas. Se discuten la importancia en la diferenciación entre las mediciones estáticas y dinámicas, y las tecnologías actuales utilizadas en cada caso. Se describen los factores relevantes que definen a un transductor y se realiza un análisis comparativo entre ellos, para luego examinar los posibles métodos de montaje, con sus beneficios y limitaciones. Finalmente se proponen distintas soluciones de bajo costo para el diseño de la sección de medición de par y velocidad sin sacrificar el rendimiento del sistema, para diferentes bancos de ensayos para controladores de máquinas eléctricas, incluyendo la electrónica de acondicionamiento de señal necesaria.

El articulo pretende ser un compendio tutorial de temas a estudiar para implementar con éxito un banco de ensayos sin dañar al transductor de par ni introducir errores de medición, ya que la información sobre esos temas está dispersa y es difícil acceder al conocimiento de los criterios de selección y procedimientos de proyecto.

Palabras clave: Máquinas eléctricas; transductor de par; medición de par.

\section{INTRODUCTION}

The importance of rotating machines in the modern world is well known. Their usefulness is evidence in almost all areas of production, industry, commerce and daily life. In order to satisfy the demands of increasingly efficient, reliable and durable machines, the ability to accurately measure the mechanical variables that determine the performance of machines such as: power, torque and angular speed is essential.

Online measurements of these quantities allow real-time monitoring, help ensure consistency in product quality, and can provide early indications of impending problems. Torque and power measurements are used to test advanced new machine designs and develop new machine components [1].

In order to perform torque sensing, various physical properties are used to convert, for example, rotations, variations in mechanical stresses, or magnetic properties into a proportional electrical signal. In general, the modification of this property is manifested in a section of the shaft on which the torque is exerted and measured.

Two types of torque transducers can be distinguished, static torque transducers, which measure torque without 
rotating or with limited angular movements, and rotary torque transducers, which rotate as part of the system and are suitable where dynamic torque measurements are required.

For speed measurement, several methods are used, these originate instruments such as mechanical or optical tachymeters, strobe lamps and tachometric dynamos, but the pulse rotary generator (encoder) is the most widely used, due to its accuracy and resolution.

The test benches developed for various powers in the Laboratory for Control of Drives, Traction and Power (LABCATYP) dependent on the Department of Electronics of the Faculty of Engineering of the University of Buenos Aires, are intended to use a technique for developments called RCP (Rapid Control Prototyping) that allows software-implemented control algorithms to interact in real time with variable drives for induction motors, application examples can be seen in [2], [3].

Traditional methods of torque measurement based on magnetic or hydraulic brakes, or dynamometric balances provide static torque measurements. Even the transducers that carry out dynamic measurements usually include a display that gives the reading of the torque value.

The development of the solutions proposed in this work is motivated by the need to have real-time torque measurement on the RPC platform to fully exploit the potential of the test bench, since the use in the control loop of this torque signal allows the developer to evaluate, compare and optimize his algorithms.

In this paper a comparative analysis of the different technologies that are currently used to measure torque, is carried out. The factors to be considered in the selection of the indicated transducer, the mounting methods and different design solutions are presented for the implementation of the mentioned benches.

\section{TYPES OF TORQUE TRANSDUCER}

There are different techniques for measuring torque, one of them is based on two toothed discs mounted on the ends of a torsion bar on which inductive, capacitive or optical sensors collect two signals whose phase will be proportional to the torsion angle of the bar and therefore to the torque [1]. Another method uses a diagonally grooved bar on its surface with two superimposed windings, one for excitation and the other for sensing, which will detect the variation in the reluctance of the grooved zone as a consequence of the torsion of the shaft [4].

However, the use of strain gauges is the most widely used method. The transducer consists of a flexible element, on which are applied gauges arranged to flex in a Wheatstone bridge configuration. The torque applied to the sensor causes tension or compression of the gauges, varying their resistance in proportion to the deformation and generating an output voltage signal proportional to the torque [5] when the bridge is energized. The benefit of using this method lies in the negligible mass of the strain gauges, which allows high accelerations and working frequencies greater than $50 \mathrm{kHz}$, ideal for both the measurement of static and dynamic torques. In combination with the properties of the bridge, it allows excellent linearity, hysteresis and repeatability characteristics, as well as a minimal effect of temperature and compensation for interference caused by parasitic pairs [6].

The static or reaction torque transducer uses the reaction torque, which is the turning force or moment, imposed on the stationary part of a device by the rotating part, as energy is delivered or absorbed. Power can be transmitted from a rotating member to a fixed one by various means, such as the magnetic field of a motor or generator, brake shoes or pads on drums or rotors, or the lubricant between a bearing and a shaft. Therefore, reaction torque sensors become useful tools for measuring properties such as engine power, braking effectiveness, lubrication and viscosity [7].

A typical example can be found with an electrodynamic balance such as the one presented in Fig. 1. A brake, whether by friction, magnetic or hydraulic, presents a resistant torque on the motor shaft, the torque produced by the motor generates an equal and opposite reaction torque in the machine casing that is transmitted to the base, it is at that fixing point where, installing the transducer by reaction, the measurement is made.

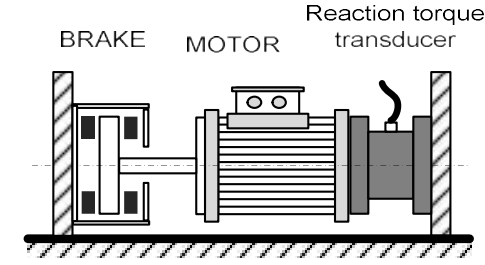

Fig. 1. Measurement of reaction torque in a motor.

Rotating or dynamic torque transducers rotate as part of the system, it can be said that they are reaction transducers that have the freedom to rotate, they acquire the torque in a rotating train of shafts, which is known as in-line torque measurement. This method allows locating the sensor as close as possible to the torque of interest and avoiding possible errors in the measurement, such as parasitic torques (bearings, etc.), extraneous loads, and components that have large rotational inertias that would dampen any dynamic torques [8]. Fig. 2 shows an example of a test bench for electrical machines.

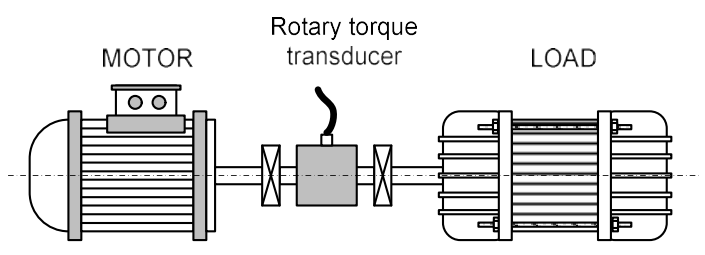

Fig. 2. Online torque measurement.

The transducers come prepared with different coupling mechanisms, the most common being: cylindrical shaft couplings and flange-type couplings. Those with a cylindrical shaft, in turn, can be smooth or with a single or double key.

In reaction transducers, the electrical connection necessary to feed the strain gauge bridge, as well as to collect the output voltage proportional to the coupling, is trivial since the sensor does not rotate. In the case of rotary transducers, there are several methods to connect the sensor mounted in the rotary application with the fixed part where the electrical connections are made. The system adopted to make this link essentially determines the performance of the transducer. 
Revista elektron, Vol. 5, No. 1, pp. 20-31 (2021)

\section{A. Slip ring transducer}

It has four conductive slip rings (composed of a copper and silver alloy, with a $90 \%$ silver content) that rotate with the sensor, and brushes that rub on the rings and provide electrical contact with the fixed part. The bridge is excited by two of the rings with the external power supply (in the order of a few Volts) and the output signal is collected through the other two (in the order of a few $\mathrm{mV}$ ). The brushes used in an instrument grade slip ring set are made of a compound of $80 \%$ silver and $20 \%$ graphite, two brushes per ring are used to ensure a positive electrical contact and the same signal quality in both directions of rotation of the shaft [9]. They are generally not recommended for continuous use or for high speeds, due to the wear of the brushes, which generates dust. This wear added to that of the rings makes periodic cleaning and replacement of brushes necessary, which discards them for use in applications where maintenance is difficult.

\section{B. Transducers with rotating transformers}

Rotary transformers provide a means of electrical connection without physical contact between the fixed part and the rotating shaft where the sensor is located. These transformers are similar to the conventional ones except that one of the windings is rotating with respect to the other, the windings are wound concentrically, with a coil rotating inside or next to another stationary coil. For these transducers, two rotary transformers are needed, one serves to transmit the excitation to the strain gauge bridge, while the second transfers the output signal to the non-rotating part of the transducer. Due to the nature of transformers, AC amplifiers are required for signal conditioning, increasing the cost of the system.

\section{Transducers with rotating analog electronics on the shaft}

This method uses rotating electronics on the shaft. As in the previous case, there is no contact between rotating and fixed parts, but in this case the power that is transmitted with a rotating transformer is rectified and stabilized on the shaft to excite the sensor. In addition, the torqueproportional output signal of the bridge is amplified and converted to variable frequency signal for transfer to the stator. A more advanced version uses a single link between rotor and stator, a fixed antenna transmits an alternating voltage of the order of $20 \mathrm{kHz}$ from the stator that is collected by a winding in the rotor, it is rectified and stabilized to feed the bridge, the output voltage from the bridge controls the frequency of the square-wave voltage, it switches a carrier of the order of $\mathrm{MHz}$ that feeds a second winding in the rotor. The antenna receives the carrier, amplifies, filters and demodulates it to recover the measurement signal [6].

\section{Transducers with rotating digital electronics on the shaft}

A more complex variant involves digitizing the torque signal on the rotor. This signal generated by the bridge is filtered, amplified and digitized, then passed to a processor that encodes it in the form of a serial word for transmission to the stator through a rotating transformer [10]. Compared to the analog signal, the measurement signal in digital form is more robust against possible disturbances. This signal enters a microprocessor and then, depending on the type of transducer, it is converted into a voltage, current or digital signal and reaches the output of the transducer where it can be measured directly at the connector.

A separate rotor feature collects the power from another transformer or other type of induction link, conditions it, and then supplies it to the other electronics in the rotor.

Other variants of these transducers use an antenna to transmit power to the rotor and telemetry through inductive or infrared coupling to transfer the signals to the stator.

\section{E. Special transducers}

The dual-range transducer is an option when the application demands a precise measurement of both the operating torque and the torque peaks, these are generally based on two bridge sensors mounted on the same axis, where the one with the lower range receives an amplification and filtering, which affects its bandwidth. Realizing a true dual range transducer is physically impossible as it would require the same torsion shaft to have two different diameters to mount each gauge bridge on and this would mean that the lower range one would be destroyed by using the higher range.

The clamp-on type transducer consists of a pre-calibrated flex bar mounted between two collars that are clamped around the shaft, the precisely spaced edges of the collars provide a measurement of shaft torque without modifying the shaft train. Naturally this transducer uses telemetry to communicate the measurement information to the fixed part.

Another alternative that manufacturers provide is the transducer with only the sensing element on the shaft, but without the signal conditioning electronics on the stator. This offers a convenient solution from the point of view of maintenance and supply of replacement for that user in a position to develop this stage.

Usually, all types of rotary transducers have a variant that includes an encoder. Convenient when there is a need to measure speed.

\section{RELEVANT FACTORS FOR THE SELECTION OF A TORQUE TRANSDUCER}

The transducer is the mechanically weakest link in the shaft train, therefore it will fail in the first instance as a consequence of a bad installation or bad choice of equipment, and this could mean an extremely costly mistake.

To correctly select the torque transducer, the specifications of the equipment must be evaluated according to the mounting environment so that the entire system is appropriate. In some cases, the installation scenario may be redesigned to adapt it to the desired characteristics of the transducer, at least in part, but this will not always be possible, so the following factors should be considered:

Torque range. The choice of the range should consider the behavior of the application. In addition to the nominal torque of the driving machine and the load, which generally represent the average value that it can deliver or receive over time, the dynamic behavior must be considered. The starting and braking couplings, as well as the pulsating couplings in the case of reciprocating motors, coupling jumps in electrical machines such as the typical star-delta starting must be observed not only for the purposes of the 
measurement but to guarantee the integrity of the equipment Typically the safe overload range is 2 times the full scale value (but the sensor life cycle is reduced) and the catastrophic overload range is 4 times that value (sensor failure). Manufacturers provide a simple calculation method to determine a safety multiplier with respect to mean torque, from the characterization of the loads and the driving machines through a service factor.

Speed range. The nominal speed of the transducer must be greater than the maximum speed of the system regardless of the direction of rotation, to ensure that it does not suffer damage. In general, the higher the torque range, the greater the diameter and weight and the lower the rotation capacity.

Accuracy and resolution. When considering measurement errors, non-linearity, hysteresis and temperature effects should be evaluated individually. If the temperature is stable during the test, the temperature errors can be ignored, in the same way if the measurements are made with increasing loads only, the hysteresis error can be ignored, and for loads measurements near the full capacity can depreciate the non-linearity [7]. If there is the possibility of correcting the data using a calibration table, the error in measurement can be minimized. An advanced user can get rid of all errors except the non-repeatability error. In relation to the resolution, the analog measurement theoretically has infinite resolution limited only by the signal-noise ratio of the system. Also, in the case of digital transducers, the resolution in bits of their analog-digital converters must be considered.

Mass and moment of inertia of the transducer. These are decisive in the stresses that the bearings must tolerate, both static, the weight they must support, and dynamic, due to the higher mass the bending vibrations of the shaft are greater. As a consequence of this, the natural frequencies of bending of the shaft will be lower. While the moment of inertia affects the angular acceleration that the system can reach and the higher its value, the lower the natural frequencies of shaft torsion.

Torsional stiffness and in other dimensions. The fact that the torque measurement is performed from the conversion of a mechanical variation to an electrical one requires the existence of a certain flexibility of the shaft. In general, flange torque transducers (method of coupling and transmission of torque) of more compact construction are more rigid in all directions than those with a cylindrical shaft [6]. A higher stiffness is related to a higher overload capacity, but at the cost of loss of resolution, it is also associated with higher natural frequencies.

Maintenance requirements. In slip-ring based transducers the brush wear must be checked and cleanliness is essential to avoid scratches on the silver rings. Although the accumulation of particles on the brushes is not desirable, care must be taken when cleaning because a thin film of graphite on the ring helps the lubrication process [9]. In the cases of non-contact torque transducers, maintenance is significantly less, especially if they do not have bearings.

Environmental factors. The application of the equipment will determine the temperature conditions to which it will be subjected, in some cases forced ventilation over heat sources or a thermal shield over the transducer and in cold environments, covers or heating enclosures must be considered. Protection against foreign bodies and fluids defined by the IP degree of the equipment must be considered to guarantee correct operation. The electromagnetic radiation from the environment must be minimized to avoid interference in the sensor electronics, the high voltage from the ignitions of internal combustion engines, large electrical machines and $\mathrm{AC}$ and $\mathrm{DC}$ variable speed drives are some typical sources of interference, a successful installation should include shielded cables and adequate grounding to improve immunity.

Torsional vibrations and in other dimensions. In addition to vibrations in the sense of measurement, parasitic, bending and axial vibrations, caused by imbalance, buckling or pulsating acceleration of masses in reciprocating motors, must be taken into account. In many cases these factors are not relevant or can be solved by adopting a simple modeling, but if the application requires greater precision or the geometry of the system is very complex, a computer is used.

\section{ADVANTAGES AND DISADVANTAGES OF EACH TECHNOLOGY}

Reaction torque sensors: Advantages of this sensor: It is suitable for any application speed. It does not require the intervention of the shaft. It does not have the problem (existing in rotating applications) of the electrical connection to the sensor and it is also a low cost solution. Disadvantages: Low dynamic response. Greater error when measuring couplings caused by its own mass.

Slip rings: As advantages can be mentioned their low inertia. Good dynamic response. Possibility of fixed or floating mounting. Economical for low torque ranges. Disadvantages: Low rigidity. Speed range limited by contact. High maintenance due to brushes and bearings. Important electrical noise with increasing speed. Low accuracy. Difficulty in measuring low ranges as a result of the drag torque generated by the brush-ring friction. Backlash, since in general it uses a shaft with a key system.

Rotary transformers: The contactless transmission stands out in this case. Low inertia. Possibility of fixed or floating mounting. The limitation of the drag torque and the high maintenance that the slip ring sensor had has disappeared. It improves the speed range, however the use of bearings and the brittleness of the transformer cores still limits the maximum RPM. Economical, but more expensive than slip rings. Disadvantages: You must use an alternating current source. Limited bandwidth. Low stiffness. Still requires bearing maintenance. Electrical noise and low accuracy, consequence of the alignment of the transformer primary and secondary. The existing air gap makes it sensitive to vibration. Backlash, since it generally uses a shaft with a key system.

Analog telemetry: It has the advantage of contactless transmission. High stiffness. The stator can be disassembled without disassembling the shaft train (versions with antenna). Very low backlash since it generally uses a flange coupling system. Low maintenance. Suitable for high speed applications. High immunity to noise. Compact and lightweight. Disadvantages: High inertia due to its larger diameter. Limited dynamic response (better than rotary transformers). Telemetry can be susceptible to interference from nearby metals.

Digital telemetry: It has the advantage of contactless transmission. High stiffness. The stator can be disassembled 
without disassembling the shaft train (versions with antenna). Very low backlash since it generally uses a flange coupling system. Low maintenance. Suitable for high speed applications. High immunity to noise. Compact and lightweight. High immunity to interference. Less uncertainty. Great bandwidth. Possibility of software configuration [11]. Disadvantages: High inertia due to its larger diameter. High cost for low torque ranges.

Clamps: The main virtue of this type of sensor is that it is not required to intervene on the shaft. Useful in applications where quick installation and uninstallation are required. It is useful for large shaft diameters. Economical for high torque ranges. High speed operating capability. Its main disadvantage is that it measures indirectly, so knowledge of the shaft characteristics and mathematical calculations are required. It has low accuracy and limited bandwidth.

\section{MECHANICAL CONSIDERATIONS FOR ASSEMBLY}

For a correct operation of the test system, the mechanical installation must meet a series of requirements that guarantee that the stresses on the transducer are kept within the limits specified by the manufacturer. If not, the measurements will be tainted by parasitic pairs, and what is worse, there is a risk of damaging the transducer [12].

These requirements include the correct selection, for each class of transducer, of the type of coupling, hardness and resistance of the coupling materials, the proper mounting method, the balancing and alignment of the rotating system.

\section{A. Compensation element}

The function of the couplings is to extend shaft transmission lines or connect sections of different shafts, whether or not they are aligned with each other [13], and they play an important role in minimizing undesirable stresses on the shafts by compensating for the inevitable geometric errors of the assembly.

Flexible single joint couplings, also called half coupling, are used to compensate for geometric errors. Static offset resulting from alignment errors or errors associated with imperfections in the components that generate off-centering of the shafts. In general, these couplings are elastic in relation to flexural deformation, but are rigid for torsional deformation. Simple joint couplings can compensate for angular misalignments, they fulfill the function of a universal joint but unlike an ideal joint, they cause restoring forces or couplings [6]. The limit of angular misalignment they allow is low, generally much less than one degree. A representation of this type of compensation element can be seen in Fig. 3(a).

Flexible double joint couplings (Fig. 3(b)), also called full coupling, can compensate for angular and parallel misalignments, they fulfill the function of two interconnected universal joints. Like simple joints, they generate restoring forces or couplings. The limit of angular and parallel misalignment they allow is low. The application of this category of couplings is limited to compensating for the minimum inaccuracies that are unavoidable in any assembly process.

Joint shafts are made up of two joints and a straight shaft between them (Fig. 3(c)), and can compensate for angular and parallel misalignments. The principle of operation is like the double joint, but does not generate restoring forces or couplings. This kind of joint allows greater displacement than the elastic elements of the couplings, which makes it possible to compensate for large angular and parallel misalignments.

(a)

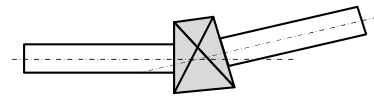

(b)

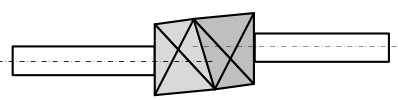

(c)

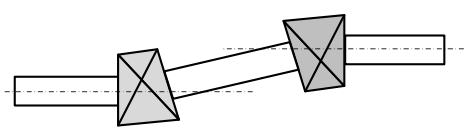

Fig. 3. (a) Single joint. (b) Double joint. (c) Joint shaft.

To select the correct coupling capacity, it is not only necessary to know the power and speed of the application, but also its severity. To determine this severity, the coupling manufacturers recommend a weighting that considers the type of load, the type of machine drive, the number of starts per hour, and the number of hours of daily operation [13].

\section{B. Types of shaft couplings}

Two large groups can be distinguished, rigid and flexible couplings.

The former are made up of pieces that rigidly join the shafts in such a way as to prevent relative movement between them. They require lubrication frequently. Some common types are plate or flange type, tapered clamp, and stud sleeve couplings (Fig. 4(a)). These are highly discouraged for the installation of torque transducers, since they cannot absorb vibrations and taking into account that both shafts must be perfectly aligned, correct operation of the transducer, or even worse, its integrity cannot be ensured.

Flexible couplings allow certain misalignments between shafts, providing the necessary compensation for geometric errors in the installation. There are a wide variety of these, which including sliding metal parts or elastic elements.

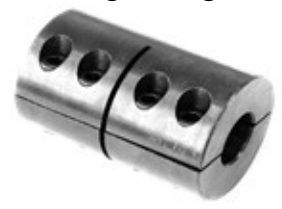

(a)

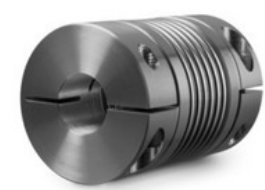

(b)
Fig. 4. (a) Rigid coupling [14]. (b) Bellows coupling [15].

Beam couplings: they are manufactured from a single piece of aluminum or stainless steel with helical grooves. They can be used as couplings with double joint functionality for low torque applications, in the order of a few tens of $\mathrm{Nm}$, have low inertia and are free of backlash. They have a relatively low torsional stiffness, due to the cuts they roll and unwind, so in demanding conditions of torque transmission, they generate positioning errors. They do not require maintenance, they do not carry lubrication. They compensate for important angular misalignments of the order of $5^{\circ}$ and radial and axial misalignments of less than $0.5 \mathrm{~mm}$.

Bellows Couplings: Consists of anodized aluminum or stainless steel hubs and stainless steel bellows, an example 
can be seen in the Fig. 4(b). They are used in motion control applications, servos, encoders, resolvers or stepper motors, due to their backlash-free operation, low inertia and high torsional rigidity. They cover torque ranges from tenths of $\mathrm{Nm}$ to a few $\mathrm{kNm}$. They do not require maintenance. Because they are totally metallic, they excel in environments with high temperatures. Their ability to absorb vibrations, in general, is inferior to couplings with polymeter inserts. They can be used as double joint, with angular compensation capacities of the order of $2^{\circ}$ and radial and axial misalignments of tenths of a $\mathrm{mm}$.

Flexible elastic band coupling: they consist of a rubber band that is mounted on the coupling hubs and fixed by means of clamping crowns and screws, (Fig. 5(a)). The coupling can be made of natural rubber or chloroprene. It is used in applications from a few $\mathrm{Nm}$ to tens of $\mathrm{kNm}$ for uneven torques, vibrations or large misalignments. It has double joint functionality with angular and radial and axial offsets of several $\mathrm{mm}$, but has very low torsional stiffness and high rotational inertia.

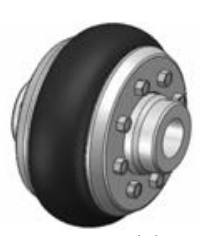

(a)

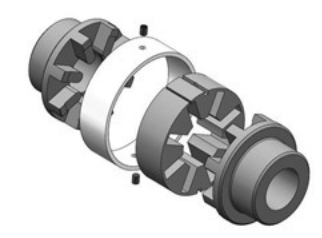

(b)
Fig. 5. (a) Flexible elastic band coupling [16]. (b) Spider coupling [16].

Star or spider couplings: it is formed by two identical cast iron cubes connected by an elastomer ring elastic to bending, but rigid to torsion, (Fig. 5(b)). The degree of torsional rigidity (soft in general) and the capacity to transmit power varies according to the material used. Nitrile is the softest, Urethane has a $50 \%$ higher power transfer capacity than Nitrile and Hytrel 200\% higher. Reliable for power transmission across the full range of services in reciprocating motor or electric machine applications. Available in a torque range of several tens of $\mathrm{kNm}$ and speeds up to $20000 \mathrm{rpm}$. They have low inertia. Its ability to compensate for misalignments is low, less than $1^{\circ}$ and tenths of an mm. It has simple joint functionality.

Universal joints: a joint is made up of two yokes connected to each other with a central cross, the movement of the drive shaft is transmitted through the cross to the driven shaft, allowing large angular misalignments of up to $25^{\circ}$ in $1800 \mathrm{rpm}$ applications. It suffers from a major problem, the transmission of speed and coupling from one shaft to the other includes an oscillatory component, which causes vibrations and wear. They require minimal lubrication. Joining two universal joints on a straight shaft (joint shaft functionality) mitigates the effect of nonuniform rotary motion. If the deflection angles of both joints are the same, the non-uniformity arising from each of the two joints is canceled and the same drive shaft torque and angular velocity occurs on the driven shaft. Although the oscillatory component is maintained in the intermediate shaft. Joint shafts are used when the design foresees that individual sections of the shaft train will be offset or move relative to each other. Also when greater compensation is required than that provided by elastic couplings. Intended for applications up to hundreds of $\mathrm{kNm}$. They have backlash. They are high cost.

Chain Couplings: These couplings consist of two hubs that include a steel sprocket each and are connected by a double chain, (Fig. 6(a)). Suitable for low speeds (due to balancing difficulties) and aggressive environments. Compact and simple. Very low inertia. Torsional stiffness limited by deformation of the chain. They require lubrication. High torque transmission capacity. They have backlash. It can compensate for angular misalignments of the order of $2^{\circ}$ but radial of tenths of an $\mathrm{mm}$.

Grid couplings: they consist of two cubes slotted on the perimeter where a serpentine-shaped steel tape is inserted, (Fig. 6(b)). The flexibility and torsional resilience of these grating couplings help reduce vibration and dampen shock loads, but provide you with reduced torsional stiffness. Torque ranges from hundreds of $\mathrm{Nm}$ to hundreds of $\mathrm{kNm}$. Limited speed. They require lubrication. Very low angular compensation capacity of less than $0.1^{\circ}$ and radial of tenths of an $\mathrm{mm}$.

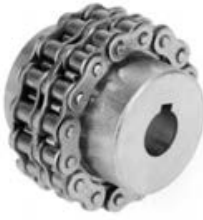

(a)

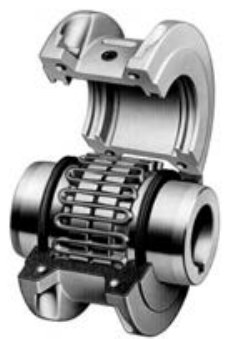

(b)
Fig. 6. (a) Chain coupling [17]. (b) Grid coupling [18].

Pin and bush couplings: Consists of two cast iron or steel hubs, connected by steel bolts sheathed with elastomer shock absorbers, (Fig. 7(a)). They are especially suitable for drives with special safety and reliability requirements. Torque shock loads and changing loads are not a problem for these flexible, compact and robust couplings. The steel variant is also particularly suitable for high-speed drives. Torque ranges higher than $\mathrm{MNm}$. They have low rotational inertia. Simple joint functionality. Angular compensation capacity less than $0.1^{\circ}$ and radial of tenths of an $\mathrm{mm}$.

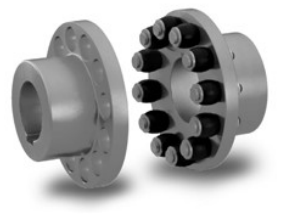

(a)

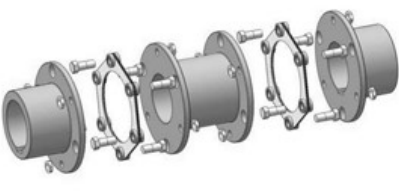

(b)
Fig. 7. (a) Pin and bush coupling [19]. (b) Steel disk coupling [16].

Steel disk couplings: These are made up of packs of thin high grade stainless steel discs. The plates are joined together by pressed bushings and are attached to the cubes with special screws. Some models are capable of spinning at high speeds (40000rpm). They can transmit high torques $(2000 \mathrm{kNm})$. They have high torsional stiffness, no backlash and low inertia. Excellent balancing characteristics. Steel multi-disc couplings require two sets of plates or plate packs to emulate the functionality of double joints or joint shafts, (Fig. 7(b)). The angular compensation allowed per pack of 
discs is up to $1^{\circ}$ in models with 6 screws $\left(0.2^{\circ}\right.$ with 10 screws) and the radial and axial compensation is a few $\mathrm{mm}$.

Gear Couplings: Each hub has external steel gear teeth that engage internal gear teeth from a flanged sleeve. The flanges are screwed together. The coupling is normally sealed. They require lubrication, except for some models made of nylon that transmit low torque. They have very low inertia and high torsional stiffness. High power density and low weight. They have wear that can cause mechanical backlash. They are capable of transmitting high torque $(\mathrm{MNm})$ with high speed. Capable of compensating angles of more than $1^{\circ}$ per gear and some $\mathrm{mm}$ of radial slippage. They can function as a double joint. They are high cost.

In all cases the specified maximum offset values cannot occur simultaneously. The allowed values are reduced if angular, parallel and axial misalignments are combined simultaneously.

\section{Geometric errors in the shaft train}

Due to the manufacturing and assembly tolerances that are always present, the various shaft train components are never fully aligned and centered with each other.

As mentioned, the transducer is the weakest element of the set and must be able to withstand all the mechanical power of the system, so any parasitic stress generated by misalignment is undesirable. To avoid or at least minimize these effects, the shaft train elements must be correctly aligned and the corresponding compensation elements used.

The system must be balanced to reduce the harmful effects of centrifugal force, which can mean forces on the transducer bearings and machines, as well as vibration. Manufacturers declare that, with careful machining of the couplings, and considering the transducer is factory balanced, no additional balancing is required for speeds below 3000rpm.

\section{SHAFT TRAIN DESIGN TO INCLUDE A TORQUE TRANSDUCER}

For the design of the shaft train a basic requirement is the static determination of the bearing configuration. This is a requirement of good mechanical design, rather than torque measurement. By definition, in mechanical engineering, a bearing configuration is statically determined when all the forces and moments on the bearings can be determined from the knowledge of the external forces and moments applying the laws of statics, without the need to determine the state of deformation [6].

There is also a requirement for kinematic determination, no shaft section or other component should move or tilt without deformation required for this to happen.

In torque measurement applications (test benches), the drive and load machine have a bearing configuration with no degrees of freedom in relation to tilting angles. A coupling of these two machines on a single shaft train can only be statically determined by inserting compensating elements with joint functionality that allow tilting movements.

On the other hand, the inclusion of a bearing element in the shaft train always requires additional couplings. The installation of torque transducers with bearings can be done in two possible ways, floating or fixed.
In floating installations the transducer is supported only by its links to the machines, through the bearings, as illustrated in Fig. 8. With the floating shaft there is only one degree of freedom between each end of the torque sensor shaft and the adjacent coupling shaft, therefore, couplings with single joint functionality are sufficient to ensure a statically determined bearing configuration. Some device, usually a flexible conductive belt, prevents the stator from rotating. This is also used to connect the transducer housing to electrical system ground.

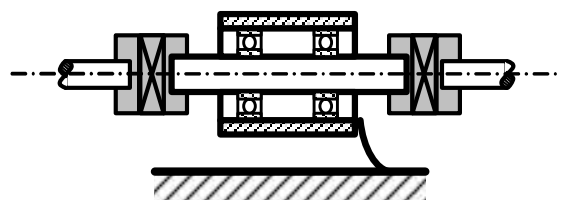

Fig. 8. Floating installation of a transducer with bearings.

This type of mounting is more tolerant of misalignment errors between the driving machine and the load, since the transducer installed between two simple joints behaves like a joint shaft, and the allowable radial misalignment is directly proportional to the distance between the points of flexion. Another advantage is the reduction of undesirable loads on the sensor, since the loads are transferred entirely through the sensor with no effect on the bearings [5].

In fixed installations the transducer is fixed to the base or bench. If the driving machine and the load are provided with bearings and supported on their legs, then couplings with double joint functionality or joint shafts are necessary to ensure a statically determined bearing configuration, this can be seen in Fig. 9.

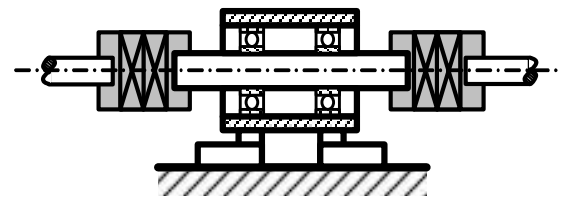

Fig. 9. Fixed installation of a transducer with bearings.

This installation reduces the mass in suspension in the couplings and can increase the critical speed of the shaft, respecting the speed rating of the torque sensor. Half of the weight of each coupling is supported by the torque sensor shaft and the other half is supported by the drive and load shafts. It is best for high rpm applications, and also for test benches where machine changes may be required, as the transducer remains supported and aligned. On the other hand, it is necessary to avoid vibrations of the transducer housing when it incorporates an encoder, to avoid errors in the speed measurement.

The disadvantage of this method is that parasitic loads on the bearings can cause heating and premature wear [5], [6].

When it comes to installing torque sensing flanges lacking bearings, single sided double joint flexible coupling or joint shaft is used. The radial and bending load due to the weight are very low in this sensor, for this reason, in general the assembly of Fig. 10 is used where the torque measuring flange supports the joint shaft. However, the load limits must always be respected with respect to parasitic loads, and if necessary add a support with bearings to support the weight of the joint. 


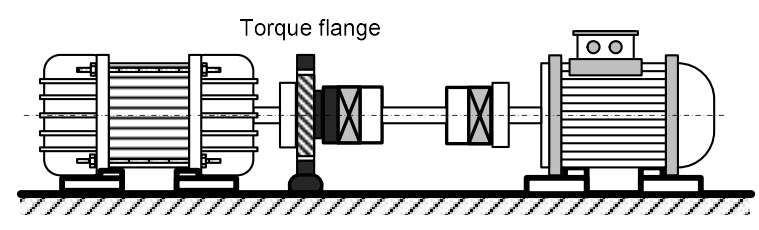

Fig. 10. Installation of a torque sensing flange.

\section{ANGULAR VELOCITY MEASUREMENT}

The importance of accurately measuring the determining mechanical variables in the performance of the machines has already been mentioned, in addition to torque, angular speed is essential in any application that involves a rotating shaft and obtaining both in real time allows to know the transferred power at all times.

According to the method used to detect a change in a physical magnitude, for example, electromagnetic radiation, magnetic field, electrical voltage or according to the type of mounting used, different types of encoders arise with different capacities, advantages and disadvantages. [20].

Mechanical: The mechanical encoder or potentiometer, limited to one or several turns according to its construction, makes it easy to obtain an absolute encoder, however, its wear and permanent adjustment limit its use.

Optical: The optical encoder, built from an electromagnetic radiation emitter, such as a diode or a laser diode, a slotted disk and at least one photo-receiver, is special for environments with a high degree of electromagnetic noise, such as for example large engines, since it is completely immune. Resolution and robustness are not an issue when properly selecting disk materials or light emitting diode. They can also be used in places with tight spaces, using their reflective variant where the beam does not pass through the slot (transmissive variant) but is reflected off the disk and is collected by the photoreceptor located in the same plane as the emitting diode. They can be used in applications where absolute or relative velocity measurements are needed, since they can be shaped according to the number of photo receptors included, being able to achieve a pseudo absolute realization using two photo-receptors and a third photo-receptor that will collect only one change per shaft turn. Among other options to select them, access to information should also be mentioned, which for different applications may be useful. In absolute encoders, the signals of all the photo-receivers can be accessed at the same time or you can also choose to obtain a single serial signal, which delays obtaining the complete data, although it also simplifies its processing because a single line of information will have to be manipulated. Other types of output for each channel can be push-pull, open-collector or line driver, depending on the cabling to be used or the system to which it will be integrated.

Magnetic: The magnetic type encoder, in contrast to its optical pair, allows it to be used in environments where there is dirt such as grease, dust, oils and even water. Basically they can be found in two large groups, magnetic or electromagnetic. The first deals with those that use a permanent magnet on the rotating axis and then one or two hall-type sensors collect the changes in the magnetic field as the movement develops. Several problems of constructive origin can occur in those of this type, the location of the hall sensor with respect to the center of rotation is important, therefore its perpendicularity also as they affect the alignment and the quality of the final measurement. Arrangements where a hall sensor that detects vertical magnetic changes is usually preferred, since it is less sensitive to the location, compared to the other type that measures magnetic field changes in the horizontal. Due to its inherently analog nature, the resolution it can achieve depends on the digitization circuit, being able to have very high resolution, accuracy and reliability performance, although for very demanding applications the optical encoder is still preferred, since when manipulating beams lower latencies are handled. Then, the second group of electromagnetic induction encoders work by detecting changes in the magnetic field between an inductor coil and another detector, depending on a disk attached to the rotor that rotates jointly with the shaft to be measured. When the disk acquires an irregular shape, the device varies the reluctance of the resulting magnetic circuit. This class is also preferred in applications where sealing is not needed, in turn its bandwidth of up to $1.5 \mathrm{MHz}$ makes it suitable for a wide variety of uses.

\section{PRACTICAL REALIZATION OF THE TORQUE AND SPEED MEASUREMENT SYSTEM (1ST SOLUTION)}

As part of a test bench for electrical machine controllers, the design and implementation of the torque and speed measurement system was carried out. In the case of torque, it was required to appreciate transients and ripples, which is why a dynamic torque transducer was chosen. Low drive machine speed and moderate utilization factor eased demands on sensor technology, this combined with a narrow torque range reduced the options to traditional sensors without electronics on the shaft. The classic slip ring transducer was chosen, favoring its superior dynamic response compared to the rotary transformer sensor, accepting the disadvantages of a higher maintenance requirement.

\section{A. Mechanical installation of torque transducer}

As a first step, the commitment made for maintenance was evaluated. Equation (1) shows an empirical formula for calculating brush life based on real field conditions and laboratory tests [21]. At $20 \%$ of the original length the spring can no longer be relied upon to hold enough tension on the brush to ensure good electrical contact with the slip ring.

$$
\text { Brush ware; Hrs to } 80 \% \text { of total }=\frac{15.5 \cdot 10^{6}}{\mathrm{RPM}},
$$

Considering that the speed of the test bench machines is around $1000 \mathrm{rpm}$, the wear of $80 \%$ of the brushes would occur after 15500 accumulated hours of operation. Time more than adequate for a laboratory application.

To define the measurement capacity of the transducer, the typical compromise was presented, on the one hand choosing a range that is too large, would mean that the accuracy and resolution might not be sufficient for the application, if instead, a range that is too low, the sensor could be damaged by overload.

To select the correct capacity, the initial step is to determine the normal running torque that should be 
measured. This can be done from the data of the characteristic plate of the bench machine, which has a nominal power of $3 \mathrm{~kW}$ at $715 \mathrm{rpm}$.

Knowing these data, the average torque in nominal operation will be:

$$
\mathrm{T}_{\text {Nom_ } 1}=\frac{\mathrm{P}}{\Omega}=\frac{3000 \mathrm{~W} \cdot 60 \mathrm{~s}}{715 \mathrm{rpm} \cdot 2 \pi}=40 \mathrm{Nm},
$$

The next step is to decide the factor of safety necessary to avoid overloading the transducer. The type of load and driving machine must be considered to determine the maximum peak torque of the system.

In general, sensor manufacturers assign a service factor to loads that is between 1 and 4, with 1 being the least severe, applied to regularly operating devices. While factor 2 is assigned to irregularly running machines or start-stop devices. Factor 3 for loads that mean big shocks and / or greater inertia. Factor 4 being the most severe and involving heavy torque reversals.

For drive machines the service factor ranges from 0 for regularly running machines to 4 for gas or diesel engines of a few pistons. The three-phase motor controlled by a frequency converter corresponds to a service factor equal to 1.

The maximum peak torque expected can be obtained by affecting the average torque by the sum of the two service factors [5]. The test bench used in this work will have as a drive machine a three-phase motor controlled by a variable frequency drive (service factor 1), and as load a direct current generator (service factor 1), this means that the maximum peak of expected torque could reach twice the average torque in nominal operation.

Considering that the priority is torque measurement with the highest accuracy and resolution (these qualities would be halved by doubling the sensor capacity), that the systems are in a controlled laboratory environment and that the sensors withstand $100 \%$ of overload without damage, a compromise was made to select a transducer with a range only $40 \%$ greater than nominal torque. A 500 lbf-in $(56.5 \mathrm{Nm})$ transducer was selected.

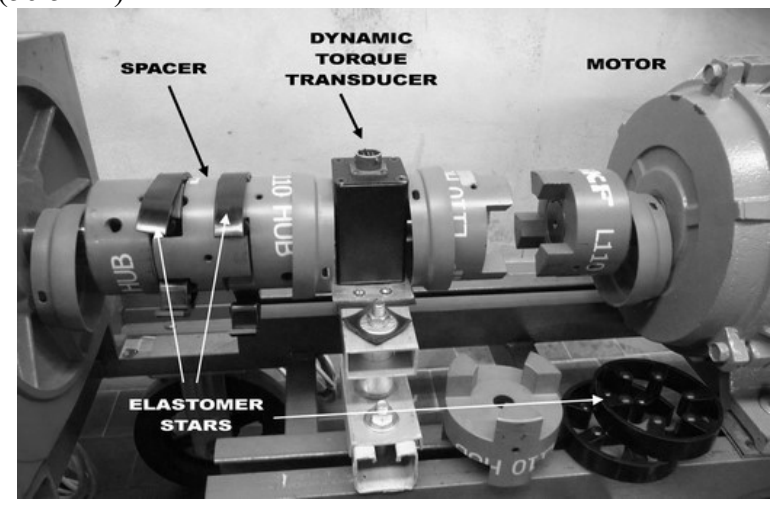

Fig. 11. Fixed mounting of the torque transducer.

In the choice of the installation method of the transducers for this work, the decision to keep vibrations on the sensor to a minimum prevailed, which is why it was decided to make a fixed installation with a double joint on both sides, also achieving the benefit of being able to change machines without having to realign the entire shaft train.
To achieve this, a design was made in our laboratory based on low-cost flexible couplings with an elastomer stars [22]. Using two additional cubes, which were turned to create two spacers and with two complementary elastomer stars, the couplings with double joint functionality were created, which ensure, for a test bench, a very adequate durability, in a simple way and at low cost (Fig. 11).

The jaw couplings mentioned provide an economical solution for standard power applications, absorbing moderate shock loads and light vibrations. The material of the elastic element is Nitrile that allows an angular misalignment of $1^{\circ}$ and parallel of $0.38 \mathrm{~mm}$ [23]. Their capacity is $105 \mathrm{Nm}$, resulting from affecting the nominal power by the average service factor corresponding to electric motors, for less than 10 hours of daily service, according to the manufacturer's manual.

\section{B. Torque signal conditioning}

The environment in which the transducer will work is subject to noise from various sources, AC and DC variable speed drives. The noise generated by this equipment radiates through the connecting cables and is coupled from the windings of the machines to the shafts and through the bearings to the housings of the machines. To minimize these effects, the machines were grounded and the electronics to condition the sensor signal were mounted in a metal box in the vicinity of the transducer. The connection was made with shielded cable and in turn the box was grounded.

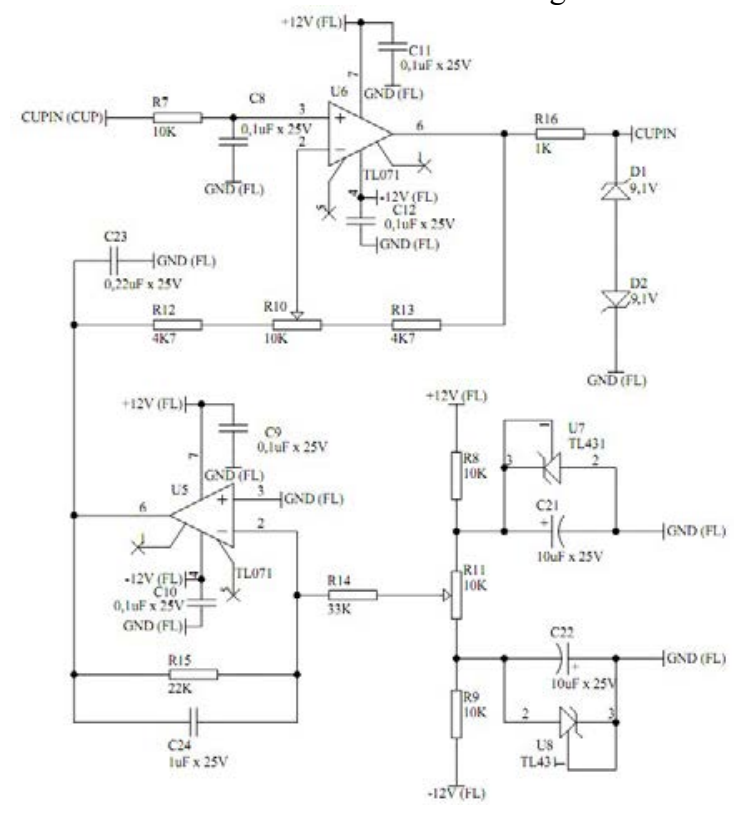

Fig. 12. Conditioning of the torque signal.

The controller test bench uses a development technique called RCP (Rapid Control Prototyping) which allows software-implemented control algorithms to interact in real time with the controlled plant hardware. A controller board housed in a PC is responsible for providing the link. The torque signal conditioner adjusts the signals delivered by the transducer to match the board. It provides offset regulation and an adjustable gain amplifier, since the transducer provides an output between $-5 \mathrm{~V}$ and $+5 \mathrm{~V}$ proportional to the coupling, CUPIN (CUP) in Fig. 12, and the board 
housed in the PC has inputs for voltages between $-10 \mathrm{~V}$ and $+10 \mathrm{~V}$ (CUPIN).

Finally, the cabling from the conditioner to the test bench was done with shielded cable to guarantee the integrity of the coupling measurement.

\section{Processing of angular velocity measurement.}

The motor-generator group includes the possibility of measuring rotation speed by various methods. One of these is based on a permanent magnet direct current generator (tachometer dynamo), another method is based on a pulse rotary generator (encoder), both are coupled to the set shaft and each generate a signal, analog in a case and digital in another, which are conditioned to use in real time on the test bench.

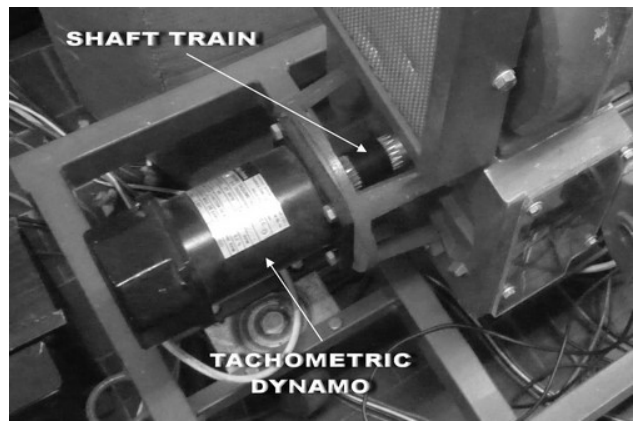

Fig. 13. Tachometric dynamo coupled to the shaft.

The analog measurement with the dynamo (Fig. 13) provides a fast responding DC voltage signal, but contains the ripple of the mechanical rectification of the collector. Being common the use of this method in the industry, it is interesting to evaluate the behavior of the tested control models when it is applied, and to compare them with the behavior that results from the use of digital measurement. The $3 \mathrm{~kW}$ bank is fitted with a $60 \mathrm{~V} / 1000 \mathrm{rpm}$ dynamo [24] with a ripple factor of $0.2 \%$.

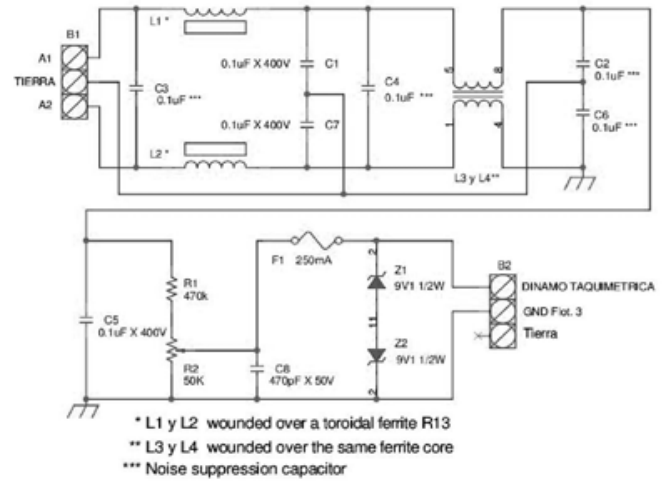

Fig. 14. Conditioning circuit for the signal from the dynamo.

In Fig. 14 you can see the conditioning circuit for the signal coming from the dynamo. It basically contains filters for electromagnetic interference, both common mode and differential mode, and an adjustable attenuator stage to scale the voltage level to that allowed by the input A / D converters of the controller board.

The digital speed measurement in this $3 \mathrm{~kW}$ set is performed by an encoder included in the dynamic torque transducer [25]. The signals delivered are conditioned by the circuit of Fig. 15 which then supplies them to the test bench where they are treated by a circuit similar to that used in solution 3 (see below in section X).
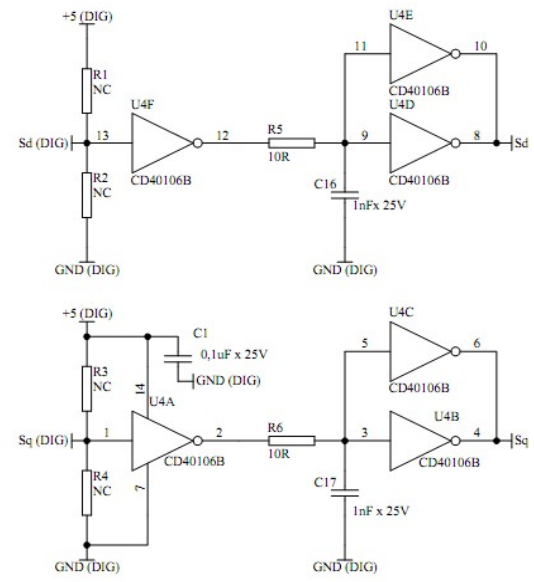

Fig. 15. Recomposition of encoder signals.

\section{PRACTICAL REALIZATION OF THE TORQUE AND SPEED MEASUREMENT SYSTEM (2ND SOLUTION)}

Using criteria similar to those mentioned in the previous case, a slip ring type transducer was selected and a fixed installation was carried out on a bench with a nominal power of the impulse machine of $1 \mathrm{CV}(736 \mathrm{~W})$ at $910 \mathrm{rpm}$, the nominal torque was compute in (3).

$$
\mathrm{T}_{\mathrm{Nom} \__{-2}}=\frac{\mathrm{P}}{\Omega}=\frac{736 \mathrm{~W} \cdot 60 \mathrm{~s}}{910 \mathrm{rpm} \cdot 2 \pi}=7.7 \mathrm{Nm},
$$

The safety factor was determined and a transducer with a range approximately $50 \%$ greater than the nominal torque was selected, a transducer of $100 \mathrm{lbf}-\mathrm{in}(11.3 \mathrm{Nm})$.

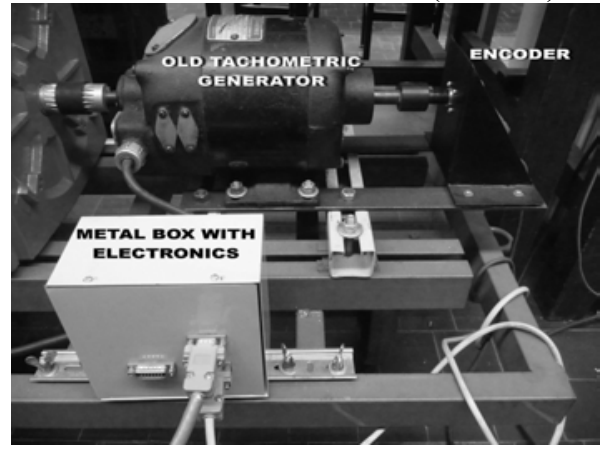

Fig. 16. Tachometric generator, encoder and metal box with electronics.

Jaw couplings were used and for installation of the transducers a fixed mounting with double joint couplings was made.

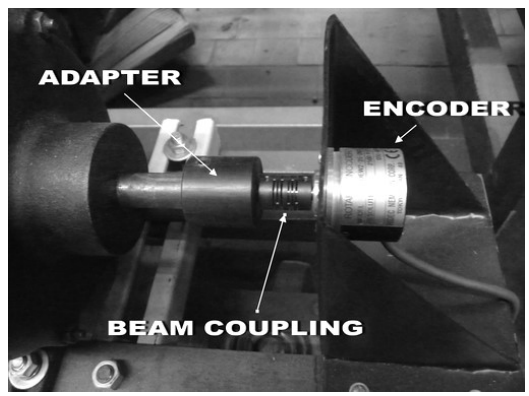

Fig. 17. Encoder with flexible beam coupling. 
The rotation speed measurement was carried out with an old tachometric generator [26] of 100V / 1000rpm. (Fig. 16) As the transducer did not include an integrated encoder, the digital speed measurement was carried out with a rotary pulse generator of $2500 \mathrm{p} / \mathrm{r}$ [27] linked by means of a flexible beam type coupling as can be seen in Fig. 17.

The electronics used are similar to that of the previous case.

\section{Practical REAlization OF THE TORQUE AND SPEED MEASUREMENT SYSTEM (3RD SOLUTION)}

This time the drive machine is a squirrel cage three-phase induction motor, $1 / 2 \mathrm{HP}, 50 \mathrm{~Hz}, 4$ poles (Siemens) of 1370rpm. The nominal coupling is determined in (4).

$$
\mathrm{T}_{\mathrm{Nom} \_3_{3}}=\frac{\mathrm{P}}{\Omega}=\frac{370 \mathrm{~W} \cdot 60 \mathrm{~s}}{1370 \mathrm{rpm} \cdot 2 \pi}=2.6 \mathrm{Nm},
$$

The torque transducer is rated at $50 \mathrm{lbf} /$ in $(5.64 \mathrm{Nm})$, max. 3000rpm (Sendev). In this case, the equipment does not include the electronics to manage the link with the sensor bridge. From the datasheet the sensitivity available is $2 \mathrm{mV} / \mathrm{V}$ at full scale [28]. In other words, for each Volt the Wheatstone bridge is supplied with, $2 \mathrm{mV}$ will be obtained at the output, if the maximum torque is applied.

Simple toothed couplings with an elastomer of intermediate hardness were used in our laboratory for assembly to allow power to be transmitted without sacrificing the measurement bandwidth [29], [30]. The installation used fixed anchor and double joint couplings as can be seen in Fig. 18 .

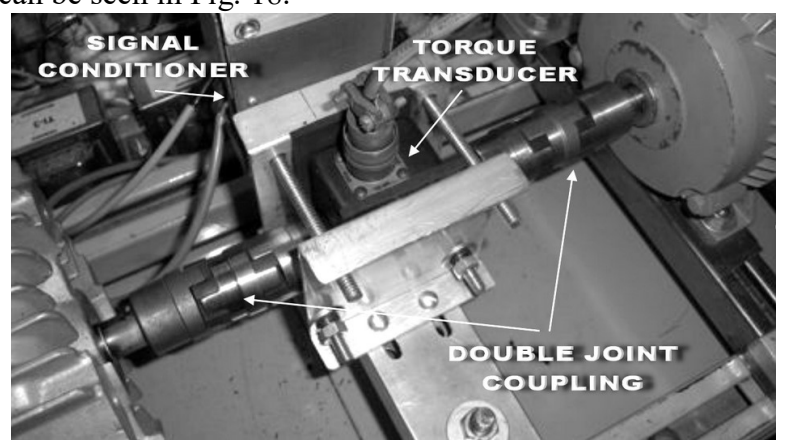

Fig. 18. Assembly with double couplings and fixed anchor.

Since the transducer signal is weak to be digitized by the driver board, a conditioner was designed to amplify it, which also supplies the voltage to energize the Wheatstone bridge. Always avoiding electromagnetic interference from the environment, which is important.

The first design factor is the location of the conditioner as close as possible to the sensor, a housing was installed inside which the entire circuit was housed. This guarantees that the path of weak signals is minimal, protecting them from possible interferences. The aforementioned casing acts as a Faraday cage that covers the entire conditioner circuit, thus protecting it from electromagnetic radiation that could disturb the operation of the circuit.

Then, despite having amplified the weak signals, the cabling to the controller's AD channel was done with shielded cable to further protect against interference.

The electronic circuit includes a dedicated power supply, which draws power from a transformer with electrostatic and electromagnetic shields, to prevent interference conducted in the power supply from affecting the measurement.

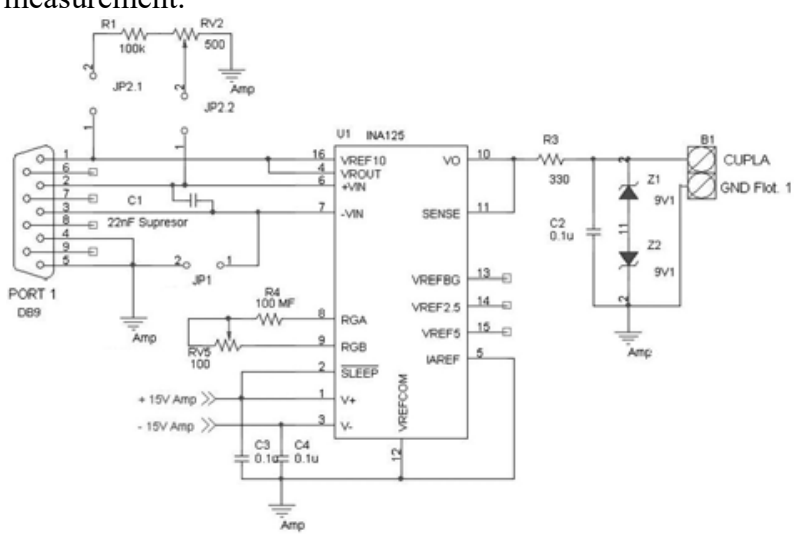

Fig. 19. Conditioner for torque signal.

The embodiment is based on an application specific monolithic integrated circuit (INA125) (see Fig. 19). The circuit provides the bridge with a stable, precise and temperature compensated power supply while its integrated instrumentation amplifier allows to modify the gain with a single adjustment. In this case the gain is adjusted by RV5 according to (5) [31].

$$
\text { Gain }=4+\frac{60 \mathrm{~K} \Omega}{\mathrm{R} 4+\mathrm{RV} 5},
$$

As a margin of safety, the conditioner gain was adjusted by simulating a signal greater than the maximum of the instrument by about $10 \%$. By varying RV5, a gain of 426 was obtained to obtain a maximum voltage of $9.5 \mathrm{~V}$ at the output. To achieve the adjustment of the conditioner gain, all the jumpers were connected and the variable resistor RV2 was regulated, previously disconnecting the transducer.

For speed, two measurements were used, an analog one similar to that addressed in the first development and a digital one from an encoder of 1024 pulses per turn.

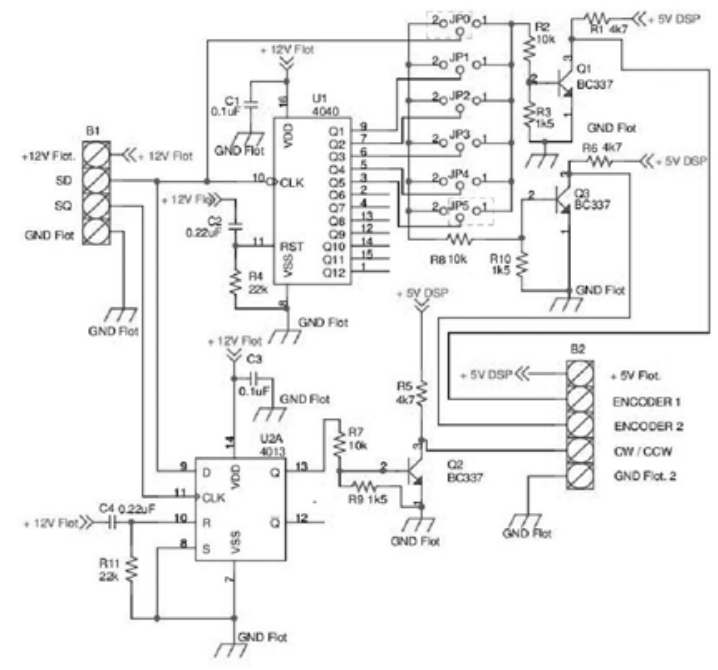

Fig. 20. Conditioner for the encoder signal.

As it is common to find in the industry measurements of rotary pulse generators of lower resolution, as can be the typical case of a gear with a magnetic sensor, a circuit 
capable of emulating the operation of said poorer measurement was made, with a divider of pulses made with a configurable binary counter so that by means of jumpers two signals can be chosen to send to the control platform, one of them is the full resolution measurement and the other is the reduced resolution one. Then, the voltage levels are adapted to those compatible with the digital inputs of the controller board (Fig. 20).

To detect the direction of rotation, a D flip-flop is used that uses the direct signal as input and the quadrature signal as clock. Thus, when the motor rotates forward, Sd leads Sq causing, on each rising edge of Sq, the flip-flop copies a logic "one" at its output. On the other hand, when turning in reverse, Sq leads Sd and therefore the output is a logical "zero".

Comparing the three solutions, it can be seen that the 1st solution is the one with the highest cost because it includes the amplification electronics and the encoder in the transducer. It requires less implementation effort and fewer resources since they are mostly mechanical realizations, electronic implementations are few. The required expertise is high. For the 2 nd solution, the cost is reduced by using an external commercial encoder, instead of purchasing a transducer with an included encoder. Increases the demand for work and resources used in assembly. The required expertise is high. In the 3 rd solution the cost is the lowest since the torque transducer only includes the sensor. The amplification and signal conditioning are user-developed. The demand for labor and resources used in the assembly are maximum. It requires a very high degree of knowledge of the subject. The dynamic response in all cases was greater than that demanded by the time constants inherent to mechanical systems.

\section{CONCLUSION}

The reliability of the results of a torque measurement and the budget allocated radically depend on the understanding of the conditions demanded by the application, accuracy, speed, bandwidth; as well as the choice of the transducer capable of satisfying them. Generally, a compromise will have to be appealed by selecting the transducer that meets the least cost.

In addition to the characteristics of the equipment, environmental factors and details of the installation must be considered. Dirt in the environment, high temperature, interference, can be some of the problems to consider. Space limitations can be decisive in the choice of transducer, as well as restrictions in mechanical mounting. The design of auxiliary electronics must always consider electromagnetic interference from the environment.

The torque transducer is the weakest link in the axle train, not addressing all the variables can mean, not only faulty measurements, but also equipment breakdown.

In this work, low-cost solutions were implemented for the design of test benches for using Rapid Control Prototyping. Various proposals for future work can be made taking advantage of this platform. Performance analysis of different PWM algorithms or study of torque disturbance recognition algorithms that make it possible to distinguish between transients and ripples are some of the possible suggestions.

\section{ACKNOWLEDGMENT}

This work has received funding from the University of Buenos Aires with funds from the subsidy UBACYT 20620170100006BA

\section{REFERENCES}

[1] I. J. Garshelis, "Torque and Power Measurement", in The Measurement, Instrumentation and Sensors Handbook, J.G. Webster (Ed.). Boca Raton: CRC Press, 1999.

[2] J. Khan, "Rapid Control Prototyping (RCP) solutions for the validation of motor control applications," 2016 International Conference on Emerging Technological Trends (ICETT), 2016, pp. 1-6, doi: 10.1109/ICETT.2016.7873699.

[3] E. Quintero-Manriquez, E. N. Sanchez, R. G. Harley, S. Li and R. A. Felix, "Neural Inverse Optimal Control Implementation for Induction Motors via Rapid Control Prototyping," in IEEE Transactions on Power Electronics, vol. 34, no. 6, pp. 5981-5992, June 2019, doi: 10.1109/TPEL.2018.2870159,

[4] Fleming, William J. "Magnetostrictive Torque Sensors Comparison of Branch, Cross, and Solenoidal Designs." SAE Transactions, vol. 99, 1990, pp. 393-420.

[5] K. Skidmore, "Torque Measurement Primer. Interface Advanced Force \& Torque Measurement, Interface Inc", 2010.

[6] R. Schicker, G. Wegener, Measuring Torque Correctly, Hottinger Baldwin Messtechnik GmbH, 2002.

[7] "Technical Information-Torque Sensor, Test \& Measurement Sensors \& Instrumentation", PCB Load \& Torque, Inc., PCB Piezotronics, 2011

[8] D. Schrand, "The Basics of Torque Measurement", Technical Notes and Articles. Sensor Development Inc., 2006.

[9] "High Quality Instrument Grade Slip Ring Assemblies A Technical Discussion”, Technote 9504/N022, SensorData Technologies, 2014.

[10] "Plug and Play" USB T25 Torque Sensor, Operation Manual, Interface, 2009.

[11] M. Minda, "How to Choose a Torque Sensor", Hottinger Brüel \& Kjaer, $2020 . \quad$ [Webinar]. Available: https://www.hbm.com/en/9016/webinar-how-to-choose-a-rotatingtorque-sensor/

[12] Rotary Torque Transducer Installation Guide, Sensor Technology, TorqSense.

[13] V. Quilodrán Jopia, “Acoplamientos Mecánicos", Ingeniería de Ejecución Mecánica en Mantenimiento Industrial, Universidad Tecnológica de Chile. INACAP.

[14] (2021) Mecapedia-Acoplamiento de manguito [Online]. Available: http://www.mecapedia.uji.es/acoplamiento_de_manguito.htm

[15] Backlash-free applications-easily solved, Siemens, 2015.

[16] Flexible couplings, Grupo Oria, 2016.

[17] Acoplamiento de cadena 10B Z16, de Gier Drive Systems.

[18] J. Piotrowski, Shaft alignment handbook, 3rd ed., CRC Press, 2006.

[19] Flexible couplings, Rupex Series, Flender Couplings, 2020.

[20] Asahi-Kasei, "Basic Knowledge of Encoder", Tutorials, Industry 4.0. [Online]. Available: www.akm.com/global/en/technology/technicaltutorial/basic-knowledge-encoder/type-mechanism-1/

[21] "Slip Ring and Slip ring Brush Maintenance", Technote 9812/N049. SensorData Technologies, 2014.

[22] A. F. Veyrat Durbex, "Cargas activas para un banco de ensayos de control de motores de inducción trifásicos," thesis, Universidad de Buenos Aires, march of 2015.

[23] Acoplamientos de mandíbula, Catalogo General, SKF.

[24] "Dínamo taquimétrica de C.C. Mocbos Modelo DT60/10, Chapa característica", Motortech

[25] "Model 01224-052, S/N 173852, Calibration data sheet", Sensor Development.

[26] "Tachometer generator, Chapa característica", General Electric.

[27] "Rotary encoder model OEW2-25-2MD, Hojas de datos", Nemicon, Nidec Nemicon Corp.

[28] “Installation - Model 01192, Data sheet", Sensor Development.

[29] Y. Nachajon, P. Witis, G. Bongiovanni, H. Tacca, and F. Ferreira, "Banco de ensayos para algoritmos de control para motores de inducción trifásicos", SAAEI, Guijón-España, 2006.

[30] Y. Nachajon Schwartz, P. Witis, and G. Bongiovanni, "Banco de ensayos para algoritmos de control para motores de inducción trifásicos", AADECA, 2006.

[31] "INA125, Data sheet", Burr-Brown Corporation, February 1997. 